Pacific Journal of Mathematics

ON GENERALIZED MOLARS OF THE PRODUCT OF
ABSTRACT HOMOGENEOUS POLYNOMIALS 


\title{
ON GENERALIZED POLARS OF THE PRODUCT OF ABSTRACT HOMOGENEOUS POLYNOMIALS
}

\author{
NEYAMAT ZAHEER
}

Let $E$ denote a vector space over an algebraically closed field $K$ of characteristic zero. Our object is to investigate the location of null-sets of generalized polars of the product of certain given abstract homogeneous polynomials from $E$ to $K$. Some special aspects of this general problem were studied in the complex plane by Bôcher and Walsh and, later, in vector spaces by Marden. Our present treatment furnishes further generalizations of the theorems of Marden, Bôcher, and Walsh and offers a systemmatic, abstract, and unified approach to their completely independent methods. One of our results, in special setting, relates to the polar of a product and reduces essentially to the author's earlier generalization [Trans. Amer. Math. Soc., 218 (1976), 115-131] of Hörmander's theorem on polars of abstract homogeneous polynomials. We show also that our theorems cannot be further generalized in certain natural directions.

1. Introduction. Let $E$ be a vector space over a field $K$ of characteristic zero. A mapping $P$ from $E$ to $K$ is called [4, pp. 760-763], [7, p. 55], [8], [14] an abstract homogeneous polynomial $(\alpha \cdot h \cdot p \cdot)$ of degree $n$ if for every $x, y \in E$,

$$
p(s x+t y)=\sum_{k=0}^{n} A_{k}(x, y) s^{k} t^{n-k} \quad \forall s, t \in K
$$

where the coefficients $A_{k}(x, y) \in K$ and are independent of $s$ and $t$ for any given $x, y$ in $E$. We shall denote by $\boldsymbol{P}_{n}$ the class of all $n$ th-degree a.h.p.'s from $E$ to $K$. The $n$ th-polar of $P$ is the mapping (see [5, Lemma 1] for its existence and uniqueness) $P\left(x_{1}, x_{2}, \cdots, x_{n}\right)$ from $E^{n}$ to $K$ which is linear in each $x_{k}$ and symmetric in the set $\left\{x_{k}\right\}$ such that $P(x, x, \cdots, x)=P(x)$ for every $x$ in $E$. The kth-polar of $P$ is then defined by

$$
P\left(x_{1}, \cdots, x_{k}, x\right)=P\left(x_{1}, \cdots, x_{k}, x, \cdots, x\right) .
$$

The null-set $Z_{P}(x, y)$ of $P$ (relative to elements $x, y$ in $E$ ) is defined [9, p. 28], [15] by

$$
Z_{P}(x, y)=\{s x+t y \neq 0 \mid s, t \in K ; P(s x+t y)=0\} .
$$

Now we shall assume throughout that $K$ is an algebraically closed field of characteristic zero. It is known [5] (see also [2, pp. 38-40], 
[11, pp. 248-255]) that $K=K_{0}(i)$, where $K_{0}$ is a maximal ordered subfield of $K$ and $-i^{2}$ is the unit element of $K$. If $z=a+i b \in K$ (with $a, b$ in $K_{0}$ ), we define $\bar{z}=a-i b, \operatorname{Re}(z)=(z+\bar{z}) / 2$ and $|z|=$ $\left(a^{2}+b^{2}\right)^{1 / 2}$. If $A \subseteq K$, we call $A$ to be $K_{0}$-convex if $\sum_{j=1}^{n} \mu_{j} a_{j} \in A$ for every $a_{j} \in A$ and $\mu_{j} \in K_{0+}$ (the set of all nonnegative elements of $K_{0}$ ) such that $\sum_{j=1}^{n} \mu_{j}=1$. Adjoin to $K$ an element $\omega$ (called infinity) and furnish $K \cup\{\omega\} \equiv K_{\omega}$ with the following structure: (1) the subset $K$ of $K_{\omega}$ preserves its initial field structure; and (2) $a+\omega=$ $\omega+a=\omega$ for every $a \in K, a \cdot \omega=\omega \cdot a=\omega$ for every $a \in K-\{0\}$, and $\omega^{-1}=0,0^{-1}=\omega$. A subset $A$ of $K_{\omega}$ is called [16, pp. 353, 373], $[14$, p. 116], [13, pp. 25-26] a generalized circular region $(g \cdot c \cdot r \cdot)$ of $K_{\omega}$ if $A$ is either one of the sets $\varnothing, K, K_{\omega}$, or $A$ satisfies the following two conditions:

(1) $\varphi_{\zeta}(A)$ is $K_{0}$-convex for every $\zeta \in K-A$, where $\varphi_{\zeta}(z)=(z-\zeta)^{-1}$ for every $z \in K_{\omega}$,

(2) $\omega \in A$ if $A$ is not $K_{0}$-convex.

The empty set $\varnothing, K, K_{\omega}$, and single-point sets (and their compliments in $\left.K_{\omega}\right)$ are examples of trivial g.c.r.'s. We shall denote by $D\left(K_{\omega}\right)$ the class of all g.c.r.'s of $K_{\omega}$. Zervos' characterization [16, pp. 372387 ] of this class, when $K$ is the field $C$ of complex numbers, leads to the following result $[16$, p. 352], $[14$, p. 116], [15], namely: The nontrivial g.c.r.'s of $\boldsymbol{C}_{\omega}$ are the open interior (or exterior) of circles or the open half-planes, adjoined with a connected subset (possibly empty) of their boundary. The g.c.r.'s of $\boldsymbol{C}_{\omega}$, with all or no boundary points included, will be called (classical) circular regions of $\boldsymbol{C}_{\omega}$.

REMARK. Through we have defined the g.c.r.'s for an algebraically closed field of characteristic zero, but the definition remains the same for any maximal ordered field $K_{0}$ (see [16, pp, 353-373], $\left[13\right.$, p. 26] for the definition of the class $D\left(K_{\omega}\right)$ when $K$ is an arbitrary field).

We now give some concepts which were introduced earlier by the author [13, pp. 36-40], [14, p. 117-119], [15] to define circular cones in $E$ and discuss some of their important properties which are found useful in later sections. Define an equivalence relation " $\sim$ " among elements of $E^{2}$ by " $(x, y) \sim\left(x^{\prime}, y^{\prime}\right)$ if and only if $\mathscr{L}[x, y]=$ $\mathscr{L}\left[x^{\prime}, y^{\prime}\right]$," where $\mathscr{L}[x, y]$ denotes the subspace of $E$ generated by the elements $x, y \in E$. The equivalence class $[(x, y)]$, containing the element $(x, y) \in E^{2}$, is called nontrivial if $x$ and $y$ are linearly independent (it is called trivial, otherwise). The axiom of choice allows us to choose a unique element from each nontrivial equivalence class. The set $N\left(\subseteq E^{2}\right)$ ef elements thus chosen would be referred to as a nucleus of $E^{2}$. Obviously, $N \neq \varnothing$ if $\operatorname{dim} E \geqq 2$. Given a nucleus $N$ of $E^{2}$ and a mapping $G: N \rightarrow D\left(K_{\omega}\right)$ (called circular mapping [14]), 
we define (cf. equations (2.1) and (2.2) in [14]) the circular cone $E_{0}(N, G)$, relative to $N$ and $G$, by

$$
E_{0}(N, G)=\bigcup T_{G}(x, y),
$$

where

$$
T_{G}(x, y)=\{s x+t y \neq 0 \mid s, t \in K ; s / t \in G(x, y)\}
$$

and the union in (1.1) ranges over all elements $(x, y) \in N$.

REMARK 1.1. If $\operatorname{dim} E=2$, then [14, Remark (2.1)] every circular cone $E_{0}(N, G)$ is of the form

$$
E_{0}(N, G)=\left\{s x_{0}+t y_{0} \neq 0 \mid s, t \in K ; s / t \in A\right\}
$$

for some $A \in D\left(K_{\omega}\right)$, where $x_{0}, y_{0}$ are any two linearly independent elements of $E$ and where $N=\left\{\left(x_{0}, y_{0}\right)\right\}$ and $G\left(x_{0}, y_{0}\right)=A$.

We define [13, p. 42], [14, p. 117], [15] hermitian cones to be subsets $E_{1}$ of $E$ of the form $E_{1}=\{x \in E \mid x \neq 0 ; H(x, x) \geqq 0\}$ (and the ones got by replacing in this expression the inequality " $\geqq$ " by " $>$ ", " $\leqq$ " or " $<$ "), where $H(x, y)$ is a hermitian symmetric form $[8, \mathrm{p}$. 270] from $E^{2}$ to $K$. For the first time, Hörmander [5] used hermitian cones in his attempt to generalize to vector spaces a theorem due to Laguerre [6], [7, Theorem $(13,2)]$ on polar derivatives and, later, Marden [8], [9] exploited these cones in generalizing to vector spaces certain classical results due to Bôcher [1], Grace [3], and to Szegö [10]. Recently, the author [13], [14], [15] succeeded in replacing the said role of hermitian cones by circular cones. The relationship between the class of hermitian cones and the class of circular cones is exhibited in the following propositions due to the author $[14, \mathrm{pp}$. 117-119]. In the rest of our work, we assume that $\operatorname{dim} E \geqq 2$.

Proposition 1.2. Let $E_{1}$ be a hermitian cone in $E$. Given a nucleus $N \subseteq E^{2}$, there exists a circular mapping $G: N \rightarrow D\left(K_{\omega}\right)$ such that $E_{0}(N, G)=E_{1}$ and $E_{1} \cap \mathscr{L}[x, y]=T_{G}(x, y)$ for every $(x, y) \in N$, where $T_{G}$ is as defined by (1.2).

Proposition 1.3. The class of all circular cones in $E$ contains properly the class of all hermitian cones.

2. A generalization of Bôcher's theorem. Before taking up our main result of this section, we shall give some definitions and useful properties. First, we establish the following proposition which expresses essentially the fact that any two circular cones can always be expressed relative to a common nucleus. 
Proposition 2.1. Given a circular cone $E_{0}(N, G)$ and an arbitrary nucleus $N^{\prime} \subseteq E^{2}$, there exists a circular mapping $G^{\prime}: N^{\prime} \rightarrow D\left(K_{\omega}\right)$ such that $E_{0}(N, G)=E_{0}\left(N^{\prime}, G^{\prime}\right)$.

Proof. From the definition of nucleus, we can define a mapping $\eta: N^{\prime} \rightarrow N$ by assigning to every element $\left(x^{\prime}, y^{\prime}\right) \in N^{\prime}$ a unique element $(x, y) \in N$ such that $(x, y) \sim\left(x^{\prime}, y^{\prime}\right)$. Then $\eta$ is a $1-1$ and onto mapping. Consequently, every element $\left(x^{\prime}, y^{\prime}\right) \in N^{\prime}$ determines uniquely an element $(x, y) \in N$, a set of scalars $\alpha, \beta, \gamma, \delta \in K$, and a homographic transformation [16, p. 353], [13, pp. 24-25] $U$ of $K_{\omega}$ such that

$$
\begin{gathered}
\eta\left(x^{\prime}, y^{\prime}\right)=(x, y), \\
x^{\prime}=\alpha x+\beta y, y^{\prime}=\gamma x+\delta y, \alpha \delta-\beta \gamma=\Delta(\text { say }) \neq 0,
\end{gathered}
$$

and

$$
U(\rho)=(\delta \rho-\gamma) /(-\beta \rho+\alpha) \forall \rho \in K_{\omega} .
$$

Let us now define $G^{\prime}\left(x^{\prime}, y^{\prime}\right)=U\left(G\left(\eta\left(x^{\prime}, y^{\prime}\right)\right)\right)=U(G(x, y))$ for every $\left(x^{\prime}, y^{\prime}\right) \in N^{\prime}$, where the element $(x, y)$ and the corresponding homographic transformation $U$ satisfies (2.1)-(2.3). Since $G(x, y) \in D\left(K_{\omega}\right)$ and since a homographic transformation permutes the class $D\left(K_{\omega}\right)$ (cf. [16, p. 353], $\left[13\right.$, p. 28]), we immediately infer that $G^{\prime}\left(x^{\prime}, y^{\prime}\right) \in D\left(K_{\omega}\right)$ and, hence, $G^{\prime}$ is indeed a circular mapping from $N^{\prime}$ into $D\left(K_{\omega}\right)$. First, we claim that

$$
E_{0}(N, G) \subseteq E_{0}\left(N^{\prime}, G^{\prime}\right) .
$$

If $z \in E_{0}(N, G)$, then there exists an element $(x, y) \in N$ and scalars $s, t \in K$ such that $z=s x+t y$ and $s / t=\rho$ (say) $\in G(x, y)$. Since $\eta$ is $1-1$ and onto, the above element $(x, y)$ of $N$ determines a unique element $\left(x^{\prime}, y^{\prime}\right) \in N^{\prime}$ and the corresponding homographic transformation $U$ satisfying the relations (2.1)-(2.3). This implies that $\left.x={\Delta^{-1}}^{-1} \delta x^{\prime}-\beta y^{\prime}\right)$, $y=\Delta^{-1}\left(\alpha y^{\prime}-\gamma x^{\prime}\right)$, and hence that

$$
z=\Delta^{-1}\left[(\delta s-\gamma t) x^{\prime}+(-\beta s+\alpha t) y^{\prime}\right]=\Delta^{-1}\left(s^{\prime} x^{\prime}+t^{\prime} y^{\prime}\right), \text { say } .
$$

Since $\rho=s / t \in G(x, y)$, the relations (2.1)-(2.3) and the definition of $G^{\prime}$ implies that $\rho^{\prime}=s^{\prime} / t^{\prime}=(\delta \rho-\gamma) /(-\beta \rho+\alpha)=U(\rho) \in G^{\prime}\left(x^{\prime}, y^{\prime}\right)$. That is, $z \in T_{G^{\prime}}\left(x^{\prime}, y^{\prime}\right) \subseteq E_{0}\left(N^{\prime}, G^{\prime}\right)$ and (2.4) holds.

Next, we claim that

$$
E_{0}\left(N^{\prime}, G^{\prime}\right) \subseteq E_{0}(N, G) .
$$

For, if $z^{\prime} \in E_{0}\left(N^{\prime}, G^{\prime}\right)$, then $z^{\prime}=s^{\prime} x^{\prime}+t^{\prime} y^{\prime}$ for some $\left(x^{\prime}, y^{\prime}\right) \in N^{\prime}$ and $s^{\prime}, t^{\prime} \in K$ such that $s^{\prime} / t^{\prime}=\rho^{\prime}($ say $) \in G^{\prime}\left(x^{\prime}, y^{\prime}\right)$. Now, $\eta$ determines uniquely an element $(x, y) \in N$, scalars $a, \beta, \gamma, \delta \in K$, and the corre- 
sponding $U$ satisfying (2.1)-(2.3). Therefore,

$$
z^{\prime}=\left(s^{\prime} \alpha+t^{\prime} \gamma\right) x+\left(s^{\prime} \beta+t^{\prime} \delta\right) y=s x+t y \quad \text { (say) }
$$

and since $s^{\prime} / t^{\prime}=\rho^{\prime} \in U(G(x, y))$, it implies that

$$
\rho=s / t=\frac{\alpha \rho^{\prime}+\gamma}{\beta \rho^{\prime}+\delta}=U^{-1}\left(\rho^{\prime}\right) .
$$

Obviously, then $\rho^{\prime}=U(\rho) \in U(G(x, y))$ and, hence, $\rho \in G(x, y)$. That is, $z^{\prime} \in T_{G}(x, y)$ and (2.5) holds. The containments (2.4) and (2.5) finally establish the desired result.

In view of the above proposition, we shall assume (without loss of generality) that all the circular cones, whenever they appear in a particular theorem, have a common nucleus.

Conventionally speaking, the word "composite (a.h.) polynomial" has been used [7, pp. 65-106], [9], [15] to designate, in general, any (a.h.) polynomial which has been derived from given (a.h.) polynomials via certain kinds of composition. In what follows we define $[8, p$. 271], [13, pp. 118-119] a special kind of composite a.h.p.'s, derived from certain given a.h.p.'s and their first polars, and study the location of the null-sets of such polynomials.

Definition 2.2. Given a.h.p.'s $P_{k} \in P_{n_{k}}$ and scalars $m_{k} \in K, k=$ $1,2, \cdots, q$, let us set

$$
\begin{aligned}
& Q(x)=P_{1}(x) \cdot P_{2}(x) \cdots P_{q}(x), \\
& Q_{k}(x)=P_{1}(x) \cdots P_{k-1}(x) \cdot P_{k+1}(x) \cdots P_{q}(x),
\end{aligned}
$$

and define

$$
\Phi\left(x_{1}, x\right)=\sum_{k=1}^{q} m_{k} Q_{k}(x) \cdot P_{k}\left(x_{1}, x\right) \forall x, x_{1} \in E .
$$

We shall call $\Phi\left(x_{1}, x\right)$ as a generalized polar of the product $Q(x)$. If $n=n_{1}+n_{2}+\cdots+n_{q}$, let us note that $Q \in \boldsymbol{P}_{n}, Q_{k} \in \boldsymbol{P}_{n-n_{k}}$ and $\boldsymbol{P}_{k}\left(x_{1}, x\right)$ is an a.h.p. of degree $n_{k}-1$ in $x$ and of degree 1 in $x_{1}, 1 \leqq k \leqq q$. Therefore, $\Phi\left(x_{1}, x\right)$ is an a.h.p. of degree $n-1$ in $x$ and an a.h.p. of degree 1 in $x_{1}$. The following proposition justifies the terminology for $\Phi\left(x_{1}, x\right)$ as "a generalized polar of $Q(x)$ ".

Proposition 2.3. In the notations of Definition 2.2, if $m_{k}=n_{k}$ for $k=1,2, \cdots, q$, then the generalized polar $\Phi\left(x_{1}, x\right)$ of the product $Q(x)$ is essentially the first polar $Q\left(x_{1}, x\right)$ of $Q(x)$, exept for a nonzero constant factor. More precisely,

$$
\Phi\left(x_{1}, x\right)=n \cdot Q\left(x_{1}, x\right) \forall x, x_{1} \in E,
$$


where $n=n_{1}+n_{2}+\cdots+n_{q}\left(m_{k}\right.$ being taken as $n_{k}$ for all $\left.k\right)$.

Proof. For each $k(1 \leqq k \leqq q)$, we use the properties of the $n_{k}$ th polar of $P_{k}$ and the fact that $K$ is algebraically closed to obtain (for every $x, x_{1} \in E$ )

$$
\begin{gathered}
P_{k}\left(s x+t x_{1}\right)=P_{k}\left(s x+t x_{1}, s x+t x_{1}, \cdots, s x+t x_{1}\right) \\
=\sum_{m=0}^{n_{k}} C\left(n_{k}, m\right) \cdot P_{k}(\overbrace{x, \cdots, x}^{m}, x_{1}, \cdots, x_{1}) \cdot s^{m} t^{n_{k}-m} \\
=\prod_{j=1}^{n_{k}}\left(\delta_{j k} \cdot s-\gamma_{j k} \cdot t\right), \text { say } \\
=\sum_{m=0}^{n_{k}}(-1)^{n_{k}-m} S(m, k) \cdot s^{m} t^{n_{k}-m},
\end{gathered}
$$

where $\delta_{j k} \equiv \delta_{j k}\left(x, x_{1}\right), \gamma_{j k} \equiv \gamma_{j k}\left(x, x_{1}\right)$ and where $S(m, k)$ denotes the sum of all possible products obtained from $\left[\delta_{1 k} \delta_{2 k} \cdots \delta_{m k} \cdot \gamma_{m+1 k} \cdots \gamma_{n_{k} k}\right]$ by permuting the subscripts $1,2, \cdots, n_{k}$ in all possible ways. The steps (2.7) and (2.9) imply that

$$
\begin{gathered}
P_{k}(x)=S\left(n_{k}, k\right)=\prod_{j=1}^{n_{k}} \delta_{j k} \\
P_{k}\left(x_{1}, x, x, \cdots, x\right) \equiv P_{k}\left(x_{1}, x\right)=-\frac{1}{n_{k}} \cdot S\left(n_{k}-1, k\right)
\end{gathered}
$$

for all $k=1,2, \cdots, q$. If we let $r_{0}=0, r_{k}=n_{1}+n_{2}+\cdots+n_{k}$ (with $r_{q}=n$ ) and define

$$
l=\psi(j, k)=r_{k-1}+j \forall j=1,2, \cdots, n_{k}, 1 \leqq k \leqq q,
$$

we easily notice that $\psi$ determines a $1-1$ correspondence between the set $\{1,2, \cdots, n\}$ and the set $\left\{(j, k) \mid 1 \leqq j \leqq n_{k} ; 1 \leqq k \leqq q\right\}$. We may then write

$$
Q\left(s x+t x_{1}\right)=\prod_{k=1}^{q} \prod_{j=1}^{n_{k}}\left(\delta_{j k} s-\gamma_{j k} t\right)=\prod_{l=1}^{n}\left(\mu_{l} s-\nu_{l} t\right), \quad \text { say },
$$

where $\mu_{l}=\delta_{j k}$ and $\nu_{l}=\gamma_{j k}$ if and only if $l=\psi(j, k)$. Next, (2.10) and (2.11) gives, respectively,

$$
Q_{k}(x)=\prod_{i=1, i \neq k}^{q}\left(\prod_{j=1}^{n_{i}} \delta_{j i}\right)=\mu_{1} \cdots \mu_{r_{k-1}} \cdot \mu_{r_{k}+1} \cdots \mu_{n}
$$




$$
\begin{aligned}
n_{k} \cdot P_{k}\left(x_{1}, x\right) & =-\sum_{m=1}^{n_{k}}\left\{\prod_{j=1, j \neq m}^{n_{k}} \delta_{j k}\right\} \cdot \gamma_{m^{k}} \\
& =-\sum_{m=1}^{n_{k}}\left\{\sum_{j=1, j \neq m}^{n_{k}} \mu_{r_{k-1}+j}\right\} \cdot \nu_{r_{k-1}+m} .
\end{aligned}
$$

Consequently, (2.14) and (2.15) imply that

$$
\begin{aligned}
n_{k} \cdot Q_{k}(x) \cdot P_{k}\left(x_{1}, x\right) & =-\sum_{m=1}^{n_{k}}\left(\mu_{1} \mu_{2} \cdots \mu_{r_{k-1}+m-1}\right) \cdot\left(\mu_{r_{k-1}+m+1} \cdots \mu_{n}\right) \nu_{r_{k-1}+m} \\
& =-\sum_{l=r_{k-1}+1}^{r_{k}}\left(\mu_{1} \mu_{2} \cdots \mu_{l-1} \cdot \nu_{l} \cdot \mu_{l+1} \cdots \mu_{n}\right) .
\end{aligned}
$$

Finally, if $m_{k}=n_{k}$ for all $k$, we obtain

$$
\begin{aligned}
\Phi\left(x_{1}, x\right) & =-\sum_{k=1}^{q}\left[\sum_{l=r_{k-1}+1}^{r_{k}}\left(\mu_{1} \cdots \mu_{l-1} \cdot \nu_{l} \cdot \mu_{l+1} \cdots \mu_{n}\right)\right] \\
& =-\sum_{l=1}^{n}\left(\mu_{1} \cdots \mu_{l-1} \cdot \nu_{l} \cdot \mu_{l+1} \cdots \mu_{n}\right) \\
& =n \cdot Q\left(x_{1}, x\right),
\end{aligned}
$$

due to the corresponding formula (2.11) for the polynomial $Q$.

REMARK 2.4. If $q=1, m_{1}=n_{1}$, the above proposition tells us that $\Phi\left(x_{1}, x\right)=n_{1} \cdot P_{1}\left(x_{1}, x\right)$.

Now we prove the following main result [13, Theorem (18.1)] of this section which generalizes a theorem due to Marden [8, Theorem (3.1)], concerning the generalized polar $\Phi\left(x_{1}, x\right)$ of the product $Q(x)$ as defined by (2.6). The complex plane version leads to certain improvements in Bôcher's theorem [1], [13, Corollary (19.3)] and in Walsh's theorem [7, Theorem (20.1)]. We prove

THEOREM 2.5. Let $E_{0}^{(i)}=E_{0}\left(N, G_{i}\right), i=1,2$, be two disjoint circular cones in $E$ and let $P_{k} \in \boldsymbol{P}_{n_{k}}(k=1,2, \cdots, q)$ such that

$$
Z_{P_{k}}(x, y) \subseteq \begin{cases}T_{G_{1}}(x, y), & k=1,2, \cdots, p(<q) \\ T_{G_{2}}(x, y), & k=p+1, \cdots, q\end{cases}
$$

for all $(x, y) \in N$. If $\Phi\left(x_{1}, x\right)$ is the generalized polar of the product $Q(x)$ (cf. Definition 2.2) with $m_{k}>0$ for $k=1,2, \cdots, p$ and $m_{k}<0$ for $k=p+1, \cdots, q$ such that

$$
\sum_{k=1}^{q} m_{k}=0
$$

then $\Phi\left(x_{1}, x\right) \neq 0$ for all linearly independent elements $x, x_{1}$ of $E$ such that $x \in E-E_{0}^{(1)} \cup E_{0}^{(2)}$. 
Proof. Let $P_{k}\left(s x+t x_{1}\right)$ be as given by the Equation (2.8) in the proof of Proposition 2.3. Let $x, x_{1}$ be linearly independent elements of $E$ such that $x \in E-E_{0}^{(1)} \cup E_{0}^{(2)}$. Then $x, x_{1}$ are nonzero elements such that $x \notin E_{0}^{(1)} \cup E_{0}^{(2)}$, so that $P_{k}(x) \neq 0$ for $1 \leqq k \leqq q$ and (due to (2.10)) $\delta_{j k} \neq 0$ for $1 \leqq j \leqq n_{k}, 1 \leqq k \leqq q$. Let $\rho_{j k}=\gamma_{j_{k}} / \delta_{j k}$. Now, $x$ and $x_{1}$ determine uniquely an element $\left(x_{0}, y_{0}\right) \in N$ and a set of scalars $\alpha, \beta, \gamma, \delta \in K$ (with $\alpha \delta-\beta \gamma \neq 0$ ) such that $\left(x_{0}, y_{0}\right) \sim\left(x, x_{1}\right)$ and such that $x=\alpha x_{0}+\beta y_{0}, x_{1}=\gamma x_{0}+\delta y_{0}$. Since (for each $k=1,2, \cdots, q$ ),

$$
P_{k}\left(\rho_{j k} x+x_{1}\right)=P_{k}\left[\left(\alpha \rho_{j k}+\gamma\right) x_{0}+\left(\beta \rho_{j k}+\delta\right) y_{0}\right]=0 \quad \forall 1 \leqq j \leqq n_{k},
$$

we see (due to (2.16)) that

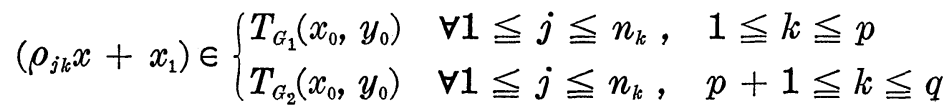

and, hence, that

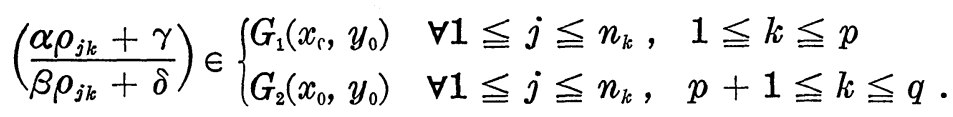

Let us put $\rho_{j k}^{\prime}=\left(\alpha \rho_{j k}+\gamma\right) /\left(\beta \rho_{j k}+\delta\right)$, so that

$$
\rho_{j k}=\left(\delta \rho_{j k}^{\prime}-\gamma\right) /\left(-\beta \rho_{j k}^{\prime}+\alpha\right)=U\left(\rho_{j k}^{\prime}\right)
$$

for all $j, k$, where $U$ is the homographic transformation given by $U(\rho)=(\delta \rho-\gamma) /(-\beta \rho+\alpha)$ for $\rho \in K_{\omega}$. That is,

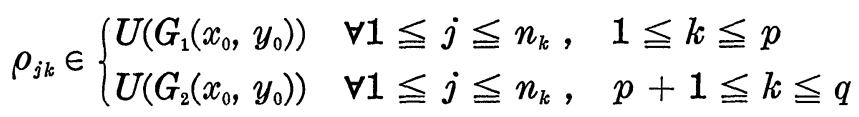

where $U\left(G_{i}\left(x_{0}, y_{0}\right)\right) \in D\left(K_{\omega}\right)$ for $i=1,2$, because $G_{i}\left(x_{0}, y_{0}\right) \in D\left(K_{\omega}\right)$ and $U$ preserves the class $D\left(K_{\omega}\right)$ (cf. [16, p. 353], [13, p. 28]). But clearly $\omega \notin U\left(G_{i}\left(x_{0}, y_{0}\right)\right)$ for $i=1,2$. For, otherwise, $\alpha / \beta \in G_{1}\left(x_{0}, y_{0}\right) U$ $G_{2}\left(x_{0}, y_{0}\right)$ (since $U(\rho)=\omega$ if and only if $\rho=\alpha / \beta$ ) and, hence, $x=\alpha x_{\mathrm{r}}+$ $\beta y_{0} \in T_{G_{1}}\left(x_{0}, y_{0}\right) \cup T_{G_{2}}\left(x_{0}, y_{0}\right) \subseteq E_{0}^{(1)} \cup E_{0}^{(2)}$, contradicting the choice of $x$ already made. Now, the definition of g.c.r. implies that the sets $U\left(G_{i}\left(x_{0}, y_{0}\right)\right), i=1,2$, are $K_{0}$-convex g.c.r.'s of $K_{\omega}$ and, hence, (2.18) implies that

$$
\sum_{j=1}^{n_{k}} \frac{1}{n_{k}} \rho_{j_{k}} \in \begin{cases}U\left(G_{1}\left(x_{0}, y_{0}\right)\right) & \forall 1 \leqq k \leqq p \\ U\left(G_{2}\left(x_{0}, y_{0}\right)\right) & \forall p+1 \leqq k \leqq q .\end{cases}
$$

If we let $A_{1}=m_{1}+m_{2}+\cdots+m_{p}$ and $A_{2}=m_{p+1}+\cdots+m_{q}$, we infer from the hypotheses on the $m_{k}$ that the scalars $m_{k} / A_{1}$ (resp. $m_{k} / A_{2}$ ) are positive elements of $K_{0}$ for $k=1,2, \cdots, p$ (resp. $k=p+1, \cdots, q$ ) with sum as 1. This fact, together with the statements (2.19) and 
the $K_{0}$-convexity of the sets $U\left(G_{i}\left(x_{0}, y_{0}\right)\right)$ for $i=1,2$, implies that $\mu_{i} / A_{i} \in U\left(G_{i}\left(x_{0}, y_{0}\right)\right)$ for $i=1,2$, where

$$
\mu_{1}=\sum_{k=1}^{p} \sum_{j=1}^{n_{k}} \frac{m_{k}}{n_{k}} \rho_{j k}, \mu_{2}=\sum_{k=p+1}^{q} \sum_{j=1}^{n_{k}} \frac{m_{k}}{n_{k}} \rho_{j k} .
$$

Therefore, there exist elements $\rho_{i} \in G_{i}\left(x_{0}, y_{0}\right), i=1,2$, such that $\mu_{i} / A_{i}=$ $U\left(\rho_{i}\right)$ for $i=1,2$, and we have

$$
\rho_{i}=\left[\left(\mu_{i} / A_{i}\right) \alpha+\gamma\right] /\left[\left(\mu_{i} / A_{i}\right) \beta+\delta\right] \in G_{i}\left(x_{0}, y_{0}\right) .
$$

That is,

$$
\left[\left(\mu_{i} / A_{i}\right) \alpha+\gamma\right] x_{0}+\left[\left(\mu_{i} / A_{i}\right) \beta+\delta\right] y_{0} \in T_{G_{i}}\left(x_{0}, y_{0}\right)
$$

and, hence, $\left(\mu_{i} / A_{i}\right) x+x_{1} \in T_{G_{i}}\left(x_{0}, y_{0}\right)$ for $i=1,2$. We claim that $\mu_{1}+\mu_{2} \neq 0$. For, otherwise, since $A_{1}+A_{2}=m_{1}+m_{2}+\cdots+m_{q}=0$, we observe that $\mu_{1} / A_{1}=\mu_{2} / A_{2}$ and that $\left(\mu_{1} / A_{1}\right) x+x_{1}$ belongs to $T_{G_{1}}\left(x_{0}, y_{0}\right) \cap T_{G_{2}}\left(x_{0}, y_{0}\right)$. That is, $\left(\mu_{1} / A_{1}\right) x+x_{1} \in E_{0}^{(1)} \cap E_{0}^{(2)}$, contradicting the hypothesis that $E_{0}^{(1)}$ and $E_{0}^{(2)}$ are disjoint. Hence

$$
\mu_{1}+\mu_{2}=\sum_{k=1}^{q} \sum_{j=1}^{n_{k}} \frac{m_{k}}{n_{k}} \cdot \rho_{j k} \neq 0 .
$$

Since $P_{k}(x) \neq 0$ for all $k$, we obtain (cf. (2.10) and (2.11))

$$
P_{k}\left(x_{1}, x\right)=-\frac{1}{n_{k}}\left(\sum_{j=1}^{n_{k}} \rho_{j k}\right) \cdot P_{k}(x) \text { for } 1 \leqq k \leqq q .
$$

Finally, since $Q_{k}(x) \cdot P_{k}(x)=Q(x) \neq 0$ for all $k$ (cf. Definition 2.2), we get (due to $(2.20)$ and (2.21))

$$
\Phi\left(x_{1}, x\right)=-\left[\sum_{k=1}^{q} \sum_{j=1}^{n_{k}} \frac{m_{k}}{n_{k}} \cdot \rho_{j k}\right] \cdot\left(\prod_{k=1}^{q} P_{k}(x)\right) \neq 0
$$

as was to be proved.

The above theorem deduces as corollary the following result due to Marden in terms of hermitian cones (a proper subclass of circular cones).

CoRollary 2.6 (Marden [8, Theorem (3.1]]). Let

$$
E_{i}=\left\{x \in E \mid x \neq 0 ; H_{i}(x, x)>0\right\}, \quad i=1,2,
$$

be two hermitian cones corresponding to the hermitian symmetric forms $H_{i}(x, y)$ from $E^{2}$ to $K$ such that $\left(E-E_{1} \cup\{0\}\right) \cap\left(E-E_{2} \cup\{0\}\right)=\varnothing$ and let $P_{k} \in P_{n_{k}}(k=1,2, \cdots, q)$ such that $P_{k}(x) \neq 0$ for $x \in E_{1}$ when $k=1,2, \cdots, p$ and such that $P_{k}(x) \neq 0$ for $x \in E_{2}$ when $k=p+1$, $p+2, \cdots, q$. If the scalars $m_{k}$ sotisfy the hypotheses of Theorem 
2.5, then $\Phi\left(x_{1}, x\right) \neq 0$ for all linearly independent elements $x, x_{1}$ of $E$ such that $x \in E_{1} \cap E_{2}$.

Proof. Starting with the hermitian cones $E-E_{i} \cup\{0\}=E_{i}^{\prime}$ (say), $i=1,2$, and taking an arbitrary nucleus $N$ of $E^{2}$, we can always get (due to Proposition 1.2) two circular mappings $G_{i}: N \rightarrow D\left(K_{\omega}\right)$ for $i=1,2$, such that $E_{i}^{\prime}=E_{0}\left(N, G_{i}\right) \equiv E_{0}^{(i)}$ (say) and such that $E_{i}^{\prime} \cap$ $\mathscr{L}[x, y]=T_{G_{i}}(x, y)$ for every $(x, y) \in N$ and $i=1,2$. We easily notice that

$$
Z_{P_{k}}(x, y) \subseteq \begin{cases}T_{G_{1}}(x, y) & \forall k=1,2, \cdots, p \\ T_{G_{2}}(x, y) & \forall k=p+1, \cdots, q\end{cases}
$$

for all $(x, y) \in N$. Since $E_{0}^{(1)}$ and $E_{0}^{(2)}$ are disjoint circular cones, all the hypotheses of Theorem 2.5 are satisfied and we conclude that $\Phi\left(x_{1}, x\right) \neq 0$ for all linearly independent elements $x, x_{1}$ of $E$ such that $x \notin E_{0}^{(1)} \cup E_{0}^{(2)}$. Since $x \neq 0$ and since $E_{0}^{(i)}=E-E_{i} \cup\{0\}$, we see that $\Phi\left(x_{1}, x\right) \neq 0$ for all linearly independent elements $x_{1}, x$ such that $x \in E_{1} \cap E_{2}$. This completes the proof.

Our second application of Theorem 2.5 gives the following corollary, which is an improved version of a theorem due to Bôcher [1], [7, Theorem (20.2)], [13, Corollary (19.3)] on the vanishing of the Jacobian of two binary forms in complex variables. The improvement is in the sense that we use g.c.r.'s, whereas Bôcher used the (classical) c.r.'s in his theorem. Our result runs as follows:

Corollary 2.7. Let $C_{1}, C_{2}$ be two disjoint g.c.r.'s of $C_{\omega}$ and let $C_{i}^{*}=\left\{(s, t) \in C^{2} \mid(s, t) \neq 0 ; s / t \in C_{i}\right), i=1,2$. If

$$
P_{i}(s, t)=\sum_{k=0}^{n} \alpha_{k i} s^{k} t^{n-k}, \quad i=1,2,
$$

are two binary forms in the complex variables $s, t$ such that all the nontrivial zeros of $P_{i}$ lie in $C_{i}^{*}$ for $i=1,2$, then all the nontrivial zeros of the Jacobian of $P_{1}$ and $P_{2}$ lie in $C_{1}^{*} \cup C_{2}^{*}$. (Note that the origin $(0,0) \in C^{2}$ is the trivial zero of every binary form).

Proof. Letting $x_{0}=(1,0), y_{0}=(0,1), N=\left\{\left(x_{0}, y_{0}\right)\right\}$, and $G_{i}\left(x_{0}, y_{0}\right)=$ $C_{i}$, we observe (cf. Remark 1.1) that the sets $C_{i}^{*}$ are precisely the disjoint circular cones $E_{0}^{(i)} \equiv E_{0}\left(N, G_{i}\right)=T_{G_{i}}\left(x_{0}, y_{0}\right)$ for $i=1,2$, and that the $P_{i}$ are basically the a.h.p.'s of degree $n$ (from $C^{2}$ to $C$ ), given by

$$
P_{i}(x) \equiv P_{i}\left(s x_{0}+t y_{0}\right)=\sum_{k=0}^{n} a_{k i} s^{k} t^{n-k} \forall x=(s, t) \in C^{2}, \quad i=1,2,
$$

such that $Z_{P_{i}}\left(x_{0} y_{0}\right) \subseteq T_{G_{i}}\left(x_{0}, y_{0}\right)$ for $i=1,2$. For all element $x=(s, t)$ 
and $x_{1}=\left(s_{1}, t_{1}\right)$ of $C^{2}$, we know [8, Equation (2.4)] that

$$
P_{i}\left(x_{1}, x\right)=\frac{1}{n}\left(s_{1} \frac{\partial P_{i}}{\partial s}+t_{1} \frac{\partial P_{i}}{\partial t}\right), \quad i=1,2,
$$

and, for $x_{1}=x$, it gives

$$
P_{i}(x)=\frac{1}{n}\left(s \frac{\partial P_{i}}{\partial s}+t \frac{\partial P_{i}}{\partial t}\right), \quad i=1,2 .
$$

If we take $q=2, p=1$, and $m_{1}=-m_{2}=1$, then $\Phi\left(x_{1}, x\right)$ in Theorem 2.5 is given by

$$
\begin{aligned}
\Phi\left(x_{1}, x\right) & =P_{1}\left(x_{1}, x\right) \cdot P_{2}(x)-P_{1}(x) \cdot P_{2}\left(x_{1}, x\right) \\
& =\frac{1}{n^{2}}\left(s_{1} t-s t_{1}\right) \cdot\left[\frac{\partial P_{1}}{\partial s} \cdot \frac{\partial P_{2}}{\partial t}-\frac{\partial P_{1}}{\partial t} \cdot \frac{\partial P_{2}}{\partial s}\right] \\
& =\frac{1}{n^{2}}\left(s_{1} t-s t_{1}\right) \cdot J(s, t), \text { say, }
\end{aligned}
$$

where $J(s, t)$ denotes the Jacobian of $P_{1}$ and $P_{2}$. Since $\Phi\left(x_{1}, x\right)$, the a.h.p.'s $P_{i}$, and the circular cones $E_{0}^{(i)}=C_{i}^{*}$ satisfy the hypotheses of Theorem 2.5, we conclude that $\Phi\left(x_{1}, x\right) \neq 0$ whenever $x, x_{1}$ are linearly independent and $x \notin C_{1}^{*} \cup C_{2}^{*}$, i.e., given any nonzero element $x=$ $(s, t) \notin C_{1}^{*} U C_{2}^{*}$, we can always choose an element $x_{1}=\left(s_{1}, t_{1}\right) \in C^{2}$ which is linearly independent to $x$ (so that $s_{1} t-s t_{1} \neq 0$ ) and for which $\Phi\left(x_{1}, x\right) \neq 0$. The equality (2.23) then says that $J(s, t) \neq 0$. Therefore, all the nontrivial zeros of the Jacobian $J(s, t)$ lie in $C_{1}^{*} U C_{2}^{*}$, as was be to proved.

If Corollary 2.7 is restated in terms of ordinary polynomials (from $C$ to $C$ ), it reduces essentially to an improved version of the second part of the two-circle theorem due to walsh [12], [7, Theorem $(20,1)]$ on the derivative of the quotient of two polynomials. The improvement is in the sense in which Corollary 2.7 improves upon Bôcher's theorem.

CoROLLARY 2.8. If all the zeros of the complex valued polynomial $f_{i}(z)$ of degree $n$ lie in the g.c.r. $C_{i}$ of $C_{\omega}(i=1,2)$ and if $C_{1} \cap C_{2}=\varnothing$, then all the finite zeros of the derivative of the quotient $f(z)=$ $f_{1}(z) / f_{2}(z)$ lie in $C_{1} \cup C_{2}$.

Proof. Let us take the sets $C_{i}^{*}$ in the manner of Corollary 2.7 and, writing $f_{i}(z)=\sum_{k=0}^{n} a_{k i} z^{k}$ for $i=1,2$, let us define

$$
P_{i}(s, t)=t^{n} \cdot f_{i}(s / t)=\sum_{k=0}^{n} a_{k i} s^{k} t^{n-k} \forall s, t \in C, \quad i=1,2 .
$$

Then the Jacobian of the binary forms $P_{1}$ and $P_{2}$ is given by (cf. [7, pp. 93-94]). 


$$
J(s, t)=n t^{2(n-1)} \cdot f^{\prime}(s / t)\left[f_{2}(s / t)\right]^{2} \quad \forall s, t \in C .
$$

Next, we notice from (2.24) that $s / t$ is a zero of $f_{i}$ if and only if $(s, t)$ is a nontrivial zero of $P_{i}$ and, from (2.25), that $s / t$ is a finite zero of $f^{\prime}$ if and only if $(s, t)$ is a nontrivial zero of $J(s, t)$. The proof is now self-evident in view of Corollary 2.7.

REMARK. Since there do exist [14, pp. 123-125] circular cones (both hermitian and otherwise) and a.h.p.'s satisfying the hypotheses of Theorem 2.5, it follows from Proposition 1.3 that our Theorem 2.5 is a strengthened generalization of Marden's theorem expressed in Corollary 2.6.

The following example shows that Theorem 2.5 cannot be generalized for vector spaces over nonalgebraically closed fields of characteristic zero.

ExAMPLE 2.9. Let $K_{0}$ be a maximal ordered field (so that $K_{0}$ is a nonalgebraically closed field of characteristic zero [11, pp. 233, 250]) and let $C_{1}=\{-1\}$ and $C_{2}=\{1\}$ be two generalized circular regions of $K_{0}$ (see Remark in $\S 1$ concerning the definition of g.c.r.'s in $\left.K_{0}\right)$. With $x_{0}=(1,0), y_{0}=(0,1)$ as basis elements of the vector space $E=K_{0}^{2}$, if we define $N=\left\{\left(x_{0}, y_{0}\right)\right\}$ and $G_{i}\left(x_{0}, y_{0}\right)=C_{i}$ for $i=1,2$, then the corresponding circular cones $E_{0}^{(i)} \equiv E_{0}\left(N, G_{i}\right)$, for $i=1,2$, are disjoint. If we take two a.h.p.'s $P_{1}, P_{2} \in P_{3}$, defined by

$$
\begin{aligned}
P_{1}(x) & \equiv P_{1}\left(s x_{0}+t y_{0}\right)=s^{3}+3 s^{2} t+3 s t^{2}+t^{3}=(s+t)^{3} \\
P_{2}(x) & \equiv P_{2}\left(s x_{0}+t y_{0}\right)=s^{3}+5 s^{2} t+4 s t^{2}-10 t^{3} \\
& =(s-t)\left[(s+3 t)^{2}+t^{2}\right]
\end{aligned}
$$

for all $x=(s, t) \in E$, then $Z_{P_{i}}\left(x_{0}, y_{0}\right) \subseteq T_{G_{i}}\left(x_{0}, y_{0}\right)$ for $i=1,2$ (since $\left[(s+3 t)^{2}+t^{2}\right]$ cannot vanish unless $s=t=0$ (cf. [1, p. 36])). Also, we know [8, Equation 2.4] that

$$
\begin{aligned}
& P_{1}\left(x_{1}, x\right)=\frac{1}{3} \cdot\left[s_{1}\left(3 s^{2}+6 s t+3 t^{2}\right)+t_{1}\left(3 s^{2}+6 s t+3 t^{2}\right)\right] \\
& P_{2}\left(x_{1}, x\right)=\frac{1}{3} \cdot\left[s_{1}\left(3 s^{2}+10 s t+4 t^{2}\right)+t_{1}\left(5 s^{2}+8 s t-30 t^{2}\right)\right]
\end{aligned}
$$

for all elements $x=(s, t)$ and $x_{1}=\left(s_{1}, t_{1}\right)$ in $E$. Let us set

$$
\Phi\left(x_{1}, x\right)=P_{1}\left(x_{1}, x\right) \cdot P_{2}(x)-P_{1}(x) \cdot P_{2}\left(x_{1}, x\right) .
$$

Now, $\Phi\left(x_{1}, x\right)$, the polynomials $P_{i}$, and the circular cones $E_{0}^{(i)}$ satisfy the hypotheses of Theorem 2.5, whereas it can be easily verified that $\Phi\left(x_{1}, x\right)=0$ for the linearly independent elements $x_{1}=(1,1)$ and $x=(1+\sqrt{69}, 2)$ in $E$, violating the conclusion in Theorem 2.5. 
Next, we ask ourselves a natural question as to whether or not the g.c.r.'s $G_{i}(x, y)$ or $C_{i}$ (employed in the hypotheses of Theorem 2.5 and Corollaries 2.7 and 2.8) can be replaced, in general, by g.c.r.'s adjoined with arbitrary subsets of their boundary, without effecting the conclusion therein. The answer is in the negative in view of the following

ExAmPle 2.10. With $E=C^{2}, K=C, x_{0}=(1,0), y_{0}=(0,1)$, and $N=\left\{\left(x_{0}, y_{0}\right)\right\}$, let us define the g.c.r.'s of $\boldsymbol{C}_{\omega}$ by

$$
G_{1}\left(x_{0}, y_{0}\right)=\left\{z \in C_{\omega} \mid \operatorname{Im}(z)>0\right\} \quad \text { and } G_{2}\left(x_{0}, y_{0}\right)=\left\{z \in \boldsymbol{C}_{\omega} \mid \operatorname{Im}(z)<0\right\}
$$

so that the corresponding circular cones $E_{0}^{(i)} \equiv E_{0}\left(N, G_{i}\right)=T_{G_{i}}\left(x_{0}, y_{0}\right)$, $i=1,2$, are disjoint. If we put $A_{1}=G_{1}\left(x_{0}, y_{0}\right) \cup\{1,2\}, A_{2}=G_{2}\left(x_{0}, y_{0}\right) \cup$ $\{-1,-2\}$, and

$$
S_{i}=\left\{s x_{0}+t y_{0} \neq 0 \mid s, t \in C ; s / t \in A_{i}\right\}, \quad i=1,2,
$$

then

$$
\begin{gathered}
S_{1}=E_{0}^{(1)} \cup\left\{s x_{0}+t y_{0} \neq 0 \mid s / t=1,2\right\}, \\
S_{2}=E_{0}^{(2)} \cup\left\{s x_{0}+t y_{0} \neq 0 \mid s / t=-1,-2\right\},
\end{gathered}
$$

so that $S_{1}, S_{2}$ (resp. $A_{1}, A_{2}$ ) are disjoint subsets of $E$ (resp. $C_{\omega}$ ) none of which are circular cones (resp. g.c.r.'s). Next, we define

$$
\begin{aligned}
& P_{1}(x) \equiv P_{1}\left(s x_{0}+t y_{0}\right)=s^{2}-3 s t+2 t^{2}=(s-t)(s-2 t), \\
& P_{2}(x) \equiv P_{2}\left(s x_{0}+t y_{0}\right)=s^{2}+3 s t+2 t^{2}=(s+t)(s+2 t),
\end{aligned}
$$

for all $x=(s, t) \in C^{2}$. Then $P_{1}, P_{2} \in P_{2}$ such that $Z_{P_{i}}\left(x_{0}, y_{0}\right) \subseteq S_{i}$ for $i=1,2$. Now, the generalized polar $\Phi\left(x_{1}, x\right)$ of $P_{1}$ and $P_{2}$, with $q=2, p=1, m_{1}=-m_{2}=+1$, is given by (cf. (2.23))

$$
\Phi\left(x_{1}, x\right)=3\left(s_{1} t-s t_{1}\right) \cdot\left(s^{2}-2 t^{2}\right)
$$

for all elements $x=(s, t)$ and $x_{1}=\left(s_{1}, t_{1}\right)$ of $E$. But, we see that $\Phi\left(x_{1}, x\right)=0$ for the linearly independent elements $x=(\sqrt{2}, 1)$ and $x_{1}=(1,1)$, where $x \notin S_{1} U S_{2}$. I.e., Theorem 2.5 no longer holds when the g.c.r.'s $G_{i}\left(x_{0}, y_{0}\right)$ are replaced, in general, by the above sets $A_{i}$.

In the language of Corollary 2.7, the above example says the following: The nontrivial zeros of the binary forms $P_{i}$ (defined above) lie in $A_{i}^{*}$ (cf. definition of $C_{i}^{*}$ in Corollary 2.7) for $i=1,2$, but the Jacobian $J(s, t)=12\left(s^{2}-2 t^{2}\right)=0$ for the element $(\sqrt{2}, 1) \notin A_{1}^{*} U A_{2}^{*}$. I.e., Corollary 2.7 does not hold, in general, when the sets $C_{i}$ are replaced by the above sets $A_{i}$. Similarly, as in passing from Corollary 2.7 to Corollary 2.8, we may express the above result in terms of ordinary 
polynomials and infer that Corollary 2.8 does not hold, in general, when the sets $C_{i}$ are replaced by the type of sets $A_{i}$ chosen above.

3. A generalization of Marden's theorem. In the previous section, we have studied the generalized polars $\Phi\left(x_{1}, x\right)$ subject to the condition that the scalar multipliers $m_{k}$ are nonzero elements of $K_{0}$ with a vanishing sum. This section primarily deals with a similar study in the case when all the $m_{k}$ 's are taken as positive. Our main theorem generalizes a result of Marden [8, Theorem (4.1)] and it involves essentially the generalization of a theorem each due to the author [14, Theorem (3.1)] and to Hörmander [5, Theorem 1]. We prove

THEOREM 3.1. Let $E_{0} \equiv E_{0}(N, G)$ be a circular cone in $E$ and let $P_{k} \in \boldsymbol{P}_{n_{k}}(k=1,2, \cdots, q)$ such that $Z_{P_{k}}(x, y) \subseteq T_{G}(x, y)$ for all $(x, y) \in$ $N$ and $k=1,2, \cdots, q$. If $\Phi\left(x_{1}, x\right)$ is a generalized polar of the product $Q(x)$ (cf. Definition 2.2) with $m_{k}>0$ for $k=1,2, \cdots, q$, then $\Phi\left(x_{1}, x\right) \neq 0$ for all nonzero elements $x, x_{1} \in E-E_{0}$.

Proof. Take any two nonzero elements $x, x_{1} \in E-E_{0}$. If $x, x_{1}$ are linearly dependent (i.e., if $x_{1}=\alpha x$ for some nonzero scalar $\alpha$ ), then $P_{k}\left(x_{1}, x\right)=P_{k}(\alpha x, x)=\alpha P_{k}(x)$ for all $k$ and hence

$$
\Phi\left(x_{1}, x\right)=\alpha\left(\sum_{k=0}^{q} m_{k}\right) \cdot \prod_{k=1}^{q} P_{k}(x) \neq 0
$$

due to the fact that $P_{k}(x) \neq 0$ for all $k$.

Now, we prove the theorem for the case when $x, x_{1}$ are linearly independent. Let

$$
P_{k}\left(s x+t x_{1}\right)=\prod_{j=1}^{n_{k}}\left(\delta_{j_{k}} s-\gamma_{j k} \cdot t\right), \quad k=1,2, \cdots, q .
$$

Since $P_{k}(x)=\delta_{1 k} \cdot \delta_{2 k} \cdots \delta_{n_{k} k} \neq 0$ and $P_{k}\left(x_{1}\right)=(-1)^{n_{k}} \cdot \gamma_{1 k} \cdot \gamma_{2 k} \cdots \gamma_{n_{k} k} \neq 0$ for all $k$, we see that $\delta_{j k}, \gamma_{j k} \neq 0$ for all $j$ and $k$. Consequently, the elements $\gamma_{j k} / \delta_{j k}=\rho_{j k}$ (say) $\neq 0$ for $1 \leqq j \leqq n_{k}$ and $1 \leqq k \leqq q$. Now, proceeding exactly as in the proof of Theorem 2.5 , we easily conclude (cf. (2.18)) that $\rho_{j k} \in U\left(G\left(x_{0}, y_{0}\right)\right)$ for all $j$ and $k$, where $\left(x_{0}, y_{0}\right) \in N$ such that $x=\alpha x_{0}+\beta y_{0}, x_{1}=\gamma x_{0}+\delta y_{0}$, and where $U(\rho)=(\delta \rho-\gamma) /(-\rho \beta+\alpha)$ for all $\rho \in K_{\omega}$. As before, $U\left(G\left(x_{0}, y_{0}\right)\right) \in D\left(K_{\omega}\right)$. Since $x, x_{1} \notin T_{G}\left(x_{0}, y_{0}\right)$, we notice that $\alpha / \beta, \gamma / \delta \notin G\left(x_{0}, y_{0}\right)$ and (hence) that $0, \omega \notin U\left(G\left(x_{0}, y_{0}\right)\right)$. That is, $U\left(G\left(x_{0}, y_{0}\right)\right)$ is a $K_{0}$-convex g.c.r. of $K_{\omega}$ which does not contain the origin. Hence, (2.19) and the succeeding arguments in the proof of Theorem 2.5 imply that $\mu / n \in U\left(G\left(x_{0}, y_{0}\right)\right)$, where $n=n_{1}+n_{2}+$ $\cdots+n_{q}$ and where 


$$
\left.\mu=\sum_{k=1}^{q} \sum_{j=1}^{n_{k}}\left(m_{k} / n_{k}\right) \cdot \rho_{j k} \neq 0 \text { (since } 0 \notin U\left(G\left(x_{0}, y_{0}\right)\right)\right) .
$$

Since $P_{k}(x) \neq 0$ for all $k$, we obtain (cf. $(2.22)$ )

$$
\Phi\left(x_{1}, x\right)=-\mu \cdot \prod_{k=1}^{q} P_{k}(x) \neq 0
$$

and the proof is complete.

The above theorem deduces as corollary the following result due to Marden and may thus be regarded (cf. Remark following Corollary 2.8) as a strengthened generalization of his theorem.

Corollary 3.2. (Marden [8, Theorem (4.1)]). Let

$$
E_{1}=\{x \in E \mid x \neq 0 ; H(x, x)>0\}
$$

be a hermitian cone in $E$, when $H(x, y)$ is a hermitian symmetric form from $E^{2}$ to $K$, and let $P_{k} \in \boldsymbol{P}_{n_{k}}(k=1,2, \cdots, q)$ such that $P_{k}(x) \neq 0$ for all $x \in E_{1}$ and $k=1,2, \cdots, q$. If $\Phi\left(x_{1}, x\right)$ is the generalized polar of the product $Q(x)$ (cf. Definition 2.2) with $m_{k}>0$ for $k=1,2, \cdots, q$, then $\Phi\left(x_{1}, x\right) \neq 0$ for all nonzero elements $x, x_{1} \in E_{1}$.

Proof. The proof is exactly similar to that of Corollary 2.6.

The following corollary is an immediate consequence of Theorem 3.1. If $q=1$, this corollary reduces essentially to the author's generalization [14, Theorem 3.1] of Laguerre's theorem, and if, in addition, $E_{0}$ is taken as a hermitian cone, it is essentially (due to Remark 2.4) a result due to Hörmander [5, Lemma 2].

Corollary 3.3. Let $E_{0} \equiv E_{0}(N, G)$ be a circular cone in $E$ and let $P_{k} \in \boldsymbol{P}_{n_{k}}(k=1,2, \cdots, q)$ such that $Z_{P_{k}}(x, y) \subseteq T_{G}(x, y)$ for all $(x, y) \in$ $N$ and $k=1,2, \cdots, q$. If $Q(x)=P_{1}(x) P_{2}(x) \cdots P_{q}(x)$ then the first polar $Q\left(x_{1}, x\right) \neq 0$ for all nonzero elements $x, x_{1} \in E-E_{0}$.

Proof. The proof is obvious in view of Proposition 2.3 and Theorem 3.1.

REMARK. In view of the examples given earlier by the author [14, p. 122], Corollary 3.3 and hence Theorem 3.1 cannot be further generalized in the two directions already discussed in case of Theorem 2.5 .

4. On two-circle theorems of Walsh. In Theorem 2.5, the circular cones $E_{0}^{(i)}(i=1,2)$ were assumed to be disjoint and the con- 
stants $m_{k}$ were taken as nonzero elements of $K_{0}$ such that $\sum_{k=1}^{q} m_{k}=$ 0 , whereas Theorem 3.1 uses only positive elements $m_{k} \in K_{0}$ (so that $\sum_{k=1}^{q} m_{k} \neq 0$ ) and utilizes only one circular cone. In this section, we study the same problem for the case when the constants $m_{k}$ are nonzero elements of $K_{0}$ such that $\sum_{k=1}^{q} m_{k} \neq 0$ and the two cones $E_{0}^{(1)}$ and $E_{0}^{(2)}$ are not necessarily disjoint. In fact we establish two main results in this section. The first one, which is somewhat like a theorem due to Marden [8, Theorem (4.2)], deduces as corollary the first part of Walsh's two-circle theorem [12], [7, Theorem (20.1)] on the critical points of rational functions. (The second part of Walsh theorem has already been considered as a corollary of Theorem 2.5.) Our second result is essentially a generalization of Walsh's two-circle theorem $[7$, Theorem $(19,1)]$ on the critical points of the product of two polynomials. Before we take up these results, we give the following definition and some relevant explanations.

Definition 4.1. Given distinct elements $\rho_{1}, \rho_{2}, \rho_{3} \in K$, we define the cross-ratio mapping (with respect to $\rho_{1}, \rho_{2}, \rho_{3}$ ) to be the homographic transformation [16, p. 353], [13, pp. 24-25] $h: K_{\omega} \rightarrow K_{\omega}$ given by

$$
h(\rho)=\frac{\rho-\rho_{2}}{\rho-\rho_{3}} \cdot \frac{\rho_{1}-\rho_{3}}{\rho_{1}-\rho_{2}}=\left(\rho, \rho_{1}, \rho_{2}, \rho_{3}\right) \quad \forall \rho \in K_{\omega} .
$$

We call $\left(\rho, \rho_{1}, \rho_{2}, \rho_{3}\right)$ as the cross-ratio of $\rho$ with $\rho_{1}, \rho_{2}, \rho_{3}$. In the case when any one of the $\rho_{i}$ 's is taken as $\omega$, we define the corresponding cross-ratio to be the expression got by deleting in (4.1) the factors which thereby involve $\omega$. E.g., $\left(p, \omega, \rho_{2}, \rho_{3}\right)=\left(\rho-\rho_{2}\right) /\left(\rho-\rho_{3}\right)$, etc.

It is trivial to verify that the homographic transformation in (4.1) maps $\rho_{1}, \rho_{2}, \rho_{3}$ to $1,0, \omega$, respectively, and that there is no other homographic transformation with this property. Consequently, identity mapping is the only homographic transformation which can map $1,0, \omega$ to $1,0, \omega$, respectively. Furthermore, cross-ratios are invariant under every homographic transformation $T$, i.e., $\left(\rho, \rho_{1}, \rho_{2}, \rho_{3}\right)=$ $\left(T \rho, T \rho_{1}, T \rho_{2}, T \rho_{3}\right)$. This follows from the fact that $T^{-1}$ is also a homographic transformation and that $h T^{-1}$ is a homographic transformation which maps $T \rho_{1}, T \rho_{2}, T \rho_{3}$ to 1,0 , $\omega$, respectively. Now we prove

THEOREM 4.2. If all the hypotheses of Theorem 2.5 are assumed, except that the circular cones $E_{0}^{(i)}(i=1,2)$ are not necessarily disjoint and that (2.17) is replaced by the condition $\sum_{k=1}^{q} m_{k} \neq 0$, and if $A_{1}=$ $\sum_{k=1}^{p} m_{k}$ and $A_{2}=\sum_{k=p+1}^{q} m_{k}$, then $\Phi\left(x_{1}, x\right) \neq 0$ for all linearly independent elements $x, x_{1}$ of $E$ such that $x_{1} \in E-E_{0}^{(1)} \cap E_{0}^{(2)}$ and $x \in E-$ $E_{0}^{(1)} \cup E_{0}^{(2)} \cup T_{S}\left(x_{0}, y_{0}\right)$, where $\left(x_{0}, y_{0}\right) \in N \cap \mathscr{L}\left[x, x_{1}\right], x_{1}=\gamma x_{0}+\delta y_{0}$, and where 


$$
S\left(x_{0}, y_{0}\right)=\left\{\rho \in K_{\omega} \mid\left(\rho, \gamma / \delta, \rho_{1}, \rho_{2}\right)=\frac{-A_{1}}{A_{2}} ; \rho_{i} \in G_{i}\left(x_{0}, y_{0}\right), i=1,2\right\}
$$

(Of course, $\Phi\left(x_{1}, x\right) \neq 0$ for any two nonzero and linearly dependent elements $x, x_{1}$ such that $x \in E-E_{0}^{(1)} \cap E_{0}^{(2)}$.)

Proof. The statement within parenthesis is self-evident in view of (3.1). In order to prove the other case, we first observe that every linearly independent pair $\left(x, x_{1}\right)$ of elements $x, x_{1} \in E$ determines a unique element $\left(x_{0}, y_{0}\right) \in N \cap \mathscr{L}\left[x, x_{1}\right]$, a unique set of scalars $\alpha, \beta, \gamma, \delta$ (with $\alpha \delta-\beta \gamma \neq 0$ ) such that $x=\alpha x_{0}+\beta y_{0}$ and $x_{1}=\gamma x_{0}+\delta y_{0}$, and, thereby, a unique set $S\left(x_{0}, y_{0}\right)$ defined by (4.2). Let us take two linearly independent elements $x, x_{1}$ of $E$ such that $x_{1} \in E-E_{0}^{(1)} \cap E_{0}^{(2)}$ and $x \in E-E_{0}^{(1)} \cup E_{0}^{(2)} \cup T_{S}\left(x_{0}, y_{0}\right)$, where $S\left(x_{0}, y_{0}\right)$ is the unique subset of $K_{\omega}$ determined in the above manner by the pair $\left(x, x_{1}\right)$. If $P_{k}\left(s x+t x_{1}\right)$ is given by (2.8), then proceeding as in the proof of Theorem $2.5 \mathrm{we}$ see that $\mu_{i} / A_{i} \in U\left(G_{i}\left(x_{0}, y_{0}\right)\right)$ for $i=1,2$, where $U(\rho)=(\delta \rho-\gamma) /(-\beta \rho+\alpha)$ for $\rho \in K_{\omega}$ and where

$$
\mu_{1}=\sum_{k=1}^{p} \sum_{j=1}^{n_{k}} \frac{m_{k}}{n_{k}} \rho_{j k} \quad \text { and } \quad \mu_{2}=\sum_{k=p+1}^{q} \sum_{j=1}^{n_{k}} \frac{m_{k}}{n_{k}} \rho_{j k} .
$$

At this point, we note that $\mu_{1}$ and $\mu_{2}$ cannot vanish simultaneously. For, otherwise, $0 \in U\left(G_{1}\left(x_{0}, y_{0}\right)\right) \cap U\left(G_{2}\left(x_{0}, y_{0}\right)\right)$ and, therefore, $\gamma / \delta$ would lie in $G_{1}\left(x_{0}, y_{0}\right) \cap G_{2}\left(x_{0}, y_{0}\right)$. This would imply that $x_{1}=\gamma x_{0}+$ $\delta y_{0} \in T_{G_{1}}\left(x_{0}, y_{0}\right) \cap T_{G_{2}}\left(x_{0}, y_{0}\right)$, contradicting the fact that $x_{1} \notin E_{0}^{(1)} \cap E_{0}^{(2)}$. Next, we observe that $\mu_{1}+\mu_{2} \neq 0$ whenever $\mu_{1}=0 \neq \mu_{2}$ or $\mu_{1} \neq$ $0=\mu_{2}$. In case, however, $\mu_{1}, \mu_{2} \neq 0$, we again show that $\mu_{1}+\mu_{2} \neq 0$ as follows: Since $\mu_{i} / A_{i}$ belongs to $U\left(G_{i}\left(x_{0}, y_{0}\right)\right)$ for $i=1,2$, there exist elements $\rho_{i} \in G_{i}\left(x_{0}, y_{0}\right)$ such that $\mu_{i} / A_{i}=U\left(\rho_{i}\right)=\left(\delta \rho_{i}-\gamma\right) /\left(-\beta \rho_{i}+\alpha\right)$ for $i=1,2$. If (on the contrary) $\mu_{1}+\mu_{2}=0$, then $\mu_{1} / \mu_{2}=-1$ and $\gamma / \delta, \rho_{1}, \rho_{2}$ are distinct elements (since $\mu_{1}, \mu_{2} \neq 0$ and $A_{1} / A_{2} \neq-1$ ) and hence

$$
\frac{\delta \rho_{2}-\gamma}{-\beta \rho_{2}+\alpha} \cdot \frac{-\beta \rho_{1}+\alpha}{\delta \rho_{1}-\gamma}=-A_{1} / A_{2} .
$$

That is (cf. Definition 4.1),

$$
\left(\alpha / \beta, \gamma / \delta, \rho_{1}, \rho_{2}\right)=\frac{\alpha / \beta-\rho_{1}}{\alpha / \beta-\rho_{2}} \cdot \frac{\gamma / \delta-\rho_{2}}{\gamma / \delta-\rho_{1}}=-A_{1} / A_{2}
$$

and, hence, $\alpha / \beta \in S\left(x_{0}, y_{0}\right)$. This implies at once that $x=\alpha x_{0}+\beta y_{0} \epsilon$ $T_{S}\left(x_{0}, y_{0}\right)$, contradicting the choice of $x$ already made above. (In the above arguments, let us note that $\beta$ and $\delta$ cannot vanish simultaneously (since $\left.A_{1} / A_{2} \neq-1\right)$.) We have, therefore, shown that in all cases $\mu_{1}+\mu_{2} \neq 0$. Finally, the proof follows from $(2.20)-(2.22)$. 
The above theorem leads to the following corollary, which is the first part of the (so-called) two-circle theorem due to Walsh on the critical points of rational functions. In the following result we shall write $D(c, r)=\{z \in C|| z-c \mid \leqq r\}$ and call it a disc with center $c$ and radius $r$.

Corollary 4.3 (Walsh [7, Theorem $(20,1)]$ ). If $f_{1}$ (resp. $f_{2}$ ) is a polynomial from $C$ to $C$ of degree $n_{1}$ (resp. $n_{2}$ ) such that all the zeros of $f_{1}$ (resp. $\left.f_{2}\right)$ lie in the disc $D\left(c_{1}, r_{1}\right) \equiv D_{1}$ (resp. $\left.D\left(c_{2}, r_{2}\right) \equiv D_{2}\right)$ and if $n_{1} \neq n_{2}$, then all the finite zeros of the derivative of the quotient $f(z)=f_{1}(z) / f_{2}(z)$ lie in $\bigcup_{i=1}^{3} D\left(c_{i}, r_{i}\right)$, where

$$
c_{3}=\frac{n_{2} c_{1}-n_{1} c_{2}}{n_{2}-n_{1}}, \quad r_{3}=\frac{n_{2} r_{1}+n_{1} r_{2}}{\left|n_{2}-n_{1}\right|}
$$

Proof. Letting $x_{0}=(1,0), y_{0}=(0,1), N=\left\{\left(x_{0}, y_{0}\right)\right\}, G_{i}\left(x_{0}, y_{0}\right)=$ $D_{i}, f_{i}(z)=\sum_{k=0}^{n_{i}} a_{k i} z^{k}$ for $i=1,2$, we notice (cf. Remark 1.1) that the sets

$$
E_{0}^{(i)} \equiv E_{0}\left(N, G_{i}\right)=\left\{s x_{0}+t y_{0} \neq 0 \mid(s, t) \in C^{2}, s / t \in D_{i}\right\}(i=1,2)
$$

are circular cones in $C^{2}$ and that the mappings $P_{i}: C^{2} \rightarrow C$, defined by

$$
P_{i}(x) \equiv P_{i}\left(s x_{0}+t y_{0}\right)=\sum_{i=0}^{n i} a_{k i} s^{k} t^{n_{i}-k} \quad \forall x=(s, t) \in C^{2}
$$

for $i=1,2$, are a.h.p.'s of degree $n_{i}$ such that $Z_{P_{i}}\left(x_{0}, y_{0}\right) \subseteq T_{G_{i}}\left(x_{0}, y_{0}\right)$ for $i=1,2$. Now the generalized polar $\Phi\left(x_{1}, x\right)$ of the product $P_{1}(x) P_{2}(x)$, given by

$$
\Phi\left(x_{1}, x\right)=n_{1} P_{1}\left(x_{1}, x\right) P_{2}(x)-n_{2} P_{1}(x) P_{2}\left(x_{1}, x\right)
$$

for all elements $x=(s, t)$ and $x_{1}=\left(s_{1}, t_{1}\right)$ of $C^{2}$, satisfies all the hypotheses of Theorem 4.2 with $m_{1}=A_{1}=n_{1}$ and $m_{2}=A_{2}=-n_{2}$. For the special choice of $x_{1}$ as $x_{0}$ (so that $s_{1}=1$ and $t_{1}=0$ ), we proceed as in the proof of Corollary 2.7 and observe that (for nonzero elements $x$ and for $i=1,2) \quad P_{i}(x)=t^{n_{i}} f_{i}(s / t) \partial P_{i} / \partial s=t^{n_{i}-1} f_{i}^{\prime}(s / t), \partial P_{i} / \partial t=$ $n_{i} t^{n_{i}-1} f_{i}(s / t)-s t^{n_{i}-2} f_{i}^{\prime}(s / t)$ and (hence) that

$$
\begin{aligned}
\Phi\left(x_{0}, x\right) & =\frac{1}{n_{1} n_{2}}\left[n_{1} \frac{\partial P_{1}}{\partial s}\left(s \frac{\partial P_{2}}{\partial s}+t \frac{\partial P_{2}}{\partial t}\right)-n_{2} \frac{\partial P_{2}}{\partial s}\left(s \frac{\partial P_{1}}{\partial s}+t \frac{\partial P_{1}}{\partial t}\right)\right] \\
& =t^{n_{1}+n_{2}-1} \cdot\left[f_{1}^{\prime}(s / t) f_{2}(s / t)-f_{1}(s / t) f_{2}^{\prime}(s / t)\right] \\
& =t^{n_{1}+n_{2}-1} \cdot f^{\prime}(s / t) \cdot\left\{f_{2}(s / t)\right\}^{2} .
\end{aligned}
$$

Since $x_{0} \notin E_{0}^{(1)} \cap E_{0}^{(2)}$, Theorem 4.2 implies that $\Phi\left(x_{0}, x\right) \neq 0$ whenever $x$ is linearly independent to $x_{0}$ such that $x \notin E_{0}^{(1)} \cup E_{0}^{(2)} \cup T_{S}\left(x_{0}, y_{0}\right)$. That is $\Phi\left(x_{0}, x\right) \neq 0$ for all elements $x=(s, t)$ for which $t \neq 0$ and 
$s / t \notin D_{1} \cup D_{2} \cup S\left(x_{0}, y_{0}\right)$, where $S\left(x_{0}, y_{0}\right)$ is given by (since $\gamma=1$ and $\delta=0$ in the notations of Theorem 4.2).

$$
\begin{aligned}
S\left(x_{0}, y_{0}\right) & =\left\{\rho \in C_{\omega} \mid\left(\rho, \omega, \rho_{1}, \rho_{2}\right)=\frac{n_{1}}{n_{2}} ; \rho_{i} \in G_{i}\left(x_{0}, y_{0}\right), i=1,2\right\} \\
& =\left\{\rho \in C_{\omega} \mid\left(\rho-\rho_{1}\right) /\left(\rho-\rho_{2}\right)=\frac{n_{1}}{n_{2}} ; \rho_{i} \in D_{i} ; i=1,2\right\} \\
& =\left\{\left(n_{2} \rho_{1}-n_{1} \rho_{2}\right) /\left(n_{2}-n_{1}\right) \mid \rho_{1} \in D_{1}, \rho_{2} \in D_{2}\right\} \\
& =D\left(c_{3}, r_{3}\right) \quad \text { (due to (4.3)). }
\end{aligned}
$$

From (4.5) it follows that $f^{\prime}(s / t) \neq 0$ for all $s, t \in C$ such that $t \neq 0$ and $s / t \notin D_{1} \cup D_{2} \cup D\left(c_{3}, r_{3}\right)$ and, hence, the corollary follows.

In the above theorem, the constants $m_{k} \in K_{0}$ have been assumed to have a nonvanishing sum, with at least one $m_{k}>0$ and at least one $m_{k}<0$. Next, we deal with a case when all the $m_{k}$ 's in Theorem 4.2 are taken as positive elements of $K_{0}$ and obtain the following corresponding result.

THEOREM 4.4. If the a.h.p.'s $P_{k}(k=1,2, \cdots, q)$ and the circular cones $E_{0}^{(1)}$ and $E_{0}^{(2)}$ (not necessarily disjoint) satisfy the conditions 2.16 of Theorem 2.5 for some $1 \leqq p<q$ and if $m_{k}>0$ for $k=1,2$, $\cdots, q$, then $\Phi\left(x_{1}, x\right) \neq 0$ for all linearly independent elements $x, x_{1}$ of $E$ such that $x_{1} \in E-E_{0}^{(1)} \cap E_{0}^{(2)}$ and $x \in E-E_{0}^{(1)} \cup E_{0}^{(2)} \cup T_{S}\left(x_{0}, y_{0}\right)$, where $S\left(x_{0}, y_{0}\right)$ is as defined in Theorem 4.2. (Of course, $\Phi\left(x_{1}, x\right) \neq 0$ whenever $x, x_{1}$ are nonzero and linearly dependent such that $x \in$ $E-E_{0}^{(1)} \cup E_{0}^{(2)}$.)

Proof. The proof is exactly the same as in Theorem 4.2.

An application of this theorem furnishes the following result on the zeros of the formal derivative of the product of two polynomials (from $K$ to $K$ ). For $K=C$, this result reduces essentially to the two-circle theorem due to Walsh [7, Theorem $(19,1)]$. By the formal derivative [16, p. 360], [14, p. 121] $f^{\prime}$ of a polynomial $f(z)=\sum_{k=0}^{n} a_{k} z^{k}$ (from $K$ to $K$ ), we mean the polynomial $f^{\prime}(z)=\sum_{k=1}^{n} k a_{k} z^{k-1}$. If, however, $P(s, t)$ is a polynomial (from $K^{2}$ to $K$ ) in $s$ and $t$, we define the formal partial derivative $\partial P / \partial s$ of $P$ with respect to $s$ (say) as the formal derivative of $P$ when $P$ is regarded as a polynomial in $s(t$ being held fixed $)$. In the following corollary, we shall write $D(c, r)=\{z \in K|| z-c \mid \leqq r\}$ and call it a ball with center $c$ (c being in $K$ ) and radius $r$ ( $r$ being in $\left.K_{0+}\right)$. The balls are usually called discs when $K=\boldsymbol{C}$. 
CoRollary 4.5. If $f_{1}$ (resp. $f_{2}$ ) is a polynomial from $K$ to $K$ of degree $n_{1}$ (resp. $n_{2}$ ) such that all the zeros of $f_{1}$ (resp. $f_{2}$ ) lie in the ball $D\left(c_{1}, r_{1}\right) \equiv D_{1}$ (resp. $\left.D\left(c_{2}, r_{2}\right) \equiv D_{2}\right)$, then all the zeros of the formal derivative of the product $f(z)=f_{1}(z) \cdot f_{2}(z)$ lie in $\bigcup_{i=1}^{3} D\left(c_{i}, r_{i}\right)$, where

$$
c_{3}=\frac{n_{1} c_{2}+n_{2} c_{1}}{n_{1}+n_{2}}, \quad r_{3}=\frac{n_{1} r_{2}+n_{2} r_{1}}{n_{1}+n_{2}}
$$

Proof. Proceeding as in the proof of Corollary 4.3, with $C$ replaced by $K$ and $\Phi\left(x_{1}, x\right)$ in (4.4) replaced by

$$
\Phi\left(x_{1}, x\right)=n_{1} P_{1}\left(x_{1}, x\right) \cdot P_{2}(x)+n_{2} P_{1}(x) \cdot P_{2}\left(x_{1}, x\right),
$$

we notice that $\Phi\left(x_{1}, x\right)$ satisfies the hypotheses of Theorem 4.4 with $m_{1}=A_{1}=n_{1}, m_{2}=A_{2}=n_{2}$. Following the computation used for obtaining (4.5), we can easily verify that (for all nonzero elements $\left.x=(s, t) \in K^{2}\right)$

$$
\begin{aligned}
\Phi\left(x_{0}, x\right) & =t^{n_{1}+n_{2}-1} \cdot\left[f_{1}^{\prime}(s / t) f_{2}(s / t)+f_{1}(s / t) \cdot f_{2}^{\prime}(s / t)\right] \\
& =t^{n_{1}+n_{2}-1} \cdot f^{\prime}(s / t),
\end{aligned}
$$

where $x_{1}=x_{0} \notin E_{0}^{(1)} \cap E_{0}^{(2)}$, (since $\omega \notin G_{i}\left(x_{0}, y_{0}\right) \equiv D_{i}$ for $i=1,2$ ) and $f_{1}^{\prime}, f_{2}^{\prime}, f^{\prime}$ denote the formal derivatives of $f_{1}, f_{2}, f$ respectively. By Theorem 4.4, $\Phi\left(x_{0}, x\right) \neq 0$ whenever the element $x=(s, t)$ is linearly independent to $x_{0}$ and is such that $x \notin E_{0}^{(1)} \cup E_{0}^{(2)} \cup T_{S}\left(x_{0}, y_{0}\right)$. That is, $f^{\prime}(s / t) \neq 0$ for all $s, t \in K$ such that $t \neq 0$ and $s / t \notin D_{1} \cup D_{2} \cup S\left(x_{0}, y_{0}\right)$, where

$$
\begin{aligned}
S\left(x_{0}, y_{0}\right) & =\left\{\rho \in K_{\omega} \mid\left(\rho, \omega, \rho_{1}, \rho_{2}\right)=-n_{1} / n_{2} ; \rho_{i} \in G_{i}\left(x_{0}, y_{0}\right), i=1,2\right\} \\
& =\left\{\rho \in K_{\omega} \mid\left(\rho-\rho_{1}\right) /\left(\rho-\rho_{2}\right)=-n_{1} / n_{2} ; \rho_{i} \in D_{i}, i=1,2\right\} \\
& =\left\{\left(n_{1} \rho_{2}+n_{2} \rho_{1}\right) /\left(n_{1}+n_{2}\right) \mid \rho_{1} \in D_{1}, \rho_{2} \in D_{2}\right\} \\
& =D\left(c_{3}, r_{3}\right) \quad \text { (due to (4.6)). }
\end{aligned}
$$

Hence, all the zeros of $f$ lie in $\mathrm{U}_{i=1}^{3} D\left(c_{i}, r_{i}\right)$, as was to be proved.

If Theorem 4.4 is specialized for the case when $G_{1}(x, y)=$ $G_{2}(x, y)=G(x, y)$ (say) for all $(x, y) \in N$ (so that $E_{0}^{(1)}=E_{0}^{(2)}=E_{0}(N, G)=$ $E_{0}$, say) we easily conclude that $\Phi\left(x_{1}, x\right) \neq 0$ for all linearly independent elements $x, x_{1}$ such that $x_{1} \in E-E_{0}$ and $x \in E-E_{0} \cup T_{S}\left(x_{0}, y_{0}\right)$, where $\left(x_{0}, y_{0}\right) \in N \cap \mathscr{L}\left[x, x_{1}\right], x_{1}=\gamma x_{0}+\delta y_{0}$ and (cf. (4.2))

$$
S\left(x_{0}, y_{0}\right)=\left\{\rho \in K_{\omega} \mid\left(\rho, \gamma / \delta, \rho_{1}, \rho_{2}\right)=-A_{1} / A_{2} ; \rho_{1}, \rho_{2} \in G\left(x_{0}, y_{0}\right)\right\} \text {. }
$$

Since $\sigma=\gamma / \delta \notin G\left(x_{0}, y_{0}\right)$ and since $\rho_{1}, \rho_{2}$ can vary over only distinct elements of $G\left(x_{0}, y_{0}\right)$, we see that every element $\rho$ of $S\left(x_{0}, y_{0}\right)$ is given by

$$
\rho=\left(A_{1} \rho_{2}+A_{2} \rho_{1}\right) /\left(A_{1}+A_{2}\right) \quad \text { if } \quad \sigma=\omega
$$


or

$$
\varphi_{\sigma}(\rho)=\left[A_{1} \varphi_{\sigma}\left(\rho_{2}\right)+A_{2} \varphi_{\sigma}\left(\rho_{1}\right)\right] /\left(A_{1}+A_{2}\right) \quad \text { if } \quad \sigma \neq \omega
$$

for some distinct elements $\rho_{1}, \rho_{2}$ in $G\left(x_{0}, y_{0}\right)$, where $\varphi_{o}(z)=1 /(z-\sigma) \forall z \epsilon$ $K_{\omega}$. In the first case, $G\left(x_{0}, y_{0}\right)$ happens to be $K_{0}$-convex (since $\omega \notin$ $\left.G\left(x_{0}, y_{0}\right)\right)$ and $\rho \in G\left(x_{0}, y_{0}\right)$. While in the second case, $\varphi_{\sigma}\left(G\left(x_{0}, y_{0}\right)\right)$ is $K_{0}$-convex (cf. definition of g.c.r.) and so $\varphi_{\sigma}(\rho) \in \varphi_{\sigma}\left(G\left(x_{0}, y_{0}\right)\right)$ and $\rho \in$ $G\left(x_{0}, y_{0}\right)$. Consequently, in either case, we discover that $S\left(x_{0}, y_{0}\right) \subseteq$ $G\left(x_{0}, y_{0}\right)$ and so $T_{S}\left(x_{0}, y_{0}\right) \subseteq T_{G}\left(x_{0}, y_{0}\right) \subseteq E_{0}$. Therefore, we conclude that $\Phi\left(x_{1}, x\right) \neq 0$ for all linearly independent elements $x, x_{1} \in E-E_{0}$. This fact together with the statement of Theorem 4.4 within parenthesis, suggests that in the present set up Theorem 4.4 reduces essentially to Theorem 3.1. In view of this and the remark following Corollary 3.3 we again notice that Theorem 4.4 and Corollary 4.5 cannot be further generalized in the two directions in which Theorem 2.5 could not be extended.

Next, we give an example to show that Theorem 4.2 cannot be generalized to vector spaces over nonalgebraically closed fields of characteristic zero.

EXAMPLE 4.6. In the notations of Example 2.9, take $E=K_{0}^{2}$, $G_{i}\left(x_{0}, y_{0}\right)=C_{i}=\{0\}$ for $i=1,2$ (so that the circular cones $E_{0}^{(1)}$ and $E_{0}^{(2)}$ are identical), and define

$$
\begin{aligned}
& P_{1}(x) \equiv P_{1}\left(s x_{0}+t y_{0}\right)=s^{3}+s t^{2}=s\left(s^{2}+t^{2}\right) \\
& P_{2}(x) \equiv P_{2}\left(s x_{0}+t y_{0}\right)=s^{3} \\
& \Phi\left(x_{1}, x\right)=2 P_{1}\left(x_{1}, x\right) \cdot P_{2}(x)-P_{1}(x) \cdot P_{2}\left(x_{1}, x\right)
\end{aligned}
$$

for all elements $x=(s, t)$ and $x_{1}=\left(s_{1}, t_{1}\right)$ of $E$. Proceeding as in Example 2.9, we can easily verify that $Z_{P_{i}}\left(x_{0}, y_{0}\right) \subseteq T_{G_{i}}\left(x_{0}, y_{0}\right)$ for $i=1,2$ and that $\Phi\left(x_{1}, x\right)=(1 / 3) s^{3}\left(3 s^{2}-t^{2}\right)$ if we take $x_{1}=x_{0}=(1,0)$. In the notations of Theorem 4.2, let us note (since $\gamma=1, \delta=0, A_{1}=$ $\left.2, A_{2}=-1\right)$ that $S\left(x_{0}, y_{0}\right)$ consists of all elements $\rho \in K_{\omega}$ such that $\left(\rho, \omega, \rho_{1}, \rho_{2}\right)=\left(\rho-\rho_{1}\right) /\left(\rho-\rho_{2}\right)=2$. That is, $S\left(x_{0}, y_{0}\right)=\varnothing$. Now, the polynomials $P_{i}$ and the generalized polar $\Phi\left(x_{1}, x\right)$ satisfy all the hypotheses of Theorem 4.2 , but $\Phi\left(x_{1}, x\right)=0$ for the linearly independent elements $x_{1}=(1,0)$ and $x=(1, \sqrt{3})$, where $x_{1} \notin E_{0}^{(1)} \cap E_{0}^{(2)}$ and $x \notin$ $E_{0}^{(1)} \cap E_{0}^{(2)} \cup T_{S}\left(x_{0}, y_{0}\right)$, contrary to the conclusion in Theorem 4.2.

Final Remark. At the end, let us recall that the condition " $E_{0}^{(1)} \cap E_{0}^{(2)}=\varnothing$ " has been used as hypothesis only in case of Theorem 2.5. In what follows, we show that this hypothesis is necessary in order for the conclusion in Theorem 2.5 to hold. To this effect, we reconsider Example 2.9 with necessary modifications: In fact, we 
replace the maximal ordered field $K_{0}$ by an algebraically closed field $K$ of characteristic zero and take the same polynomials $P_{1}(x), P_{2}(x)$, $\Phi\left(x_{1}, x\right)$ and the same g.c.r. $C_{1}=G_{1}\left(x_{0}, y_{0}\right)$ as in Example 2.9, but this time we define

$$
\begin{aligned}
C_{2}=G_{2}\left(x_{0}, y_{0}\right) & =\left\{z \in K_{\omega}|| 8 z+9 \mid \leqq 17\right\} \\
& =\left\{z \in K_{\omega}|| 64 z \bar{z}+72(z+\bar{z})-208 \leqq 0\right\}
\end{aligned}
$$

Indeed, $C_{2} \in D\left(K_{\omega}\right)[14$, p. 116] and the elements $z= \pm 1$ and $z=$ $-3 \pm i$ belong to $C_{2}$, so that $C_{1} \cap C_{2}=\{-1\}$ and (hence) $E_{0}^{(1)} \cap E_{0}^{(2)} \neq$ $\varnothing$. Also $Z_{P_{i}}\left(x_{0}, y_{0}\right) \subseteq T_{G_{i}}\left(x_{0}, y_{0}\right)$ for $i=1,2$. Therefore, all the hypotheses of Theorem 2.5 are satisfied by the polynomials $P_{1}(x)$, $P_{2}(x), \Phi\left(x_{1}, x\right)$ and the circular cones $E_{0}^{(1)}, E_{0}^{(2)}$ (except that they are disjoint), whereas $\Phi\left(x_{1}, x\right)=0$ (see Example 2.9) for the linearly independent elements $x_{1}=(1,1)$ and $x=(1+\sqrt{69}, \mid 2) \notin E_{0}^{(1)} \cup E_{0}^{(2)}$ (since $\left.(1+\sqrt{69}) / 2 \notin C_{2}\right)$. This is contrary to the conclusion in Theorem 2.5.

\section{REFERENCES}

1. M. Bôcher, $A$ problem in Statics and its relation to certain algebraic invariants, Proc. Amer. Acad. Arts Sci., 40 (1904), 469-484.

2. N. Bourbaki, Éléments de mathématique. XIV. Part 1, Let structures fondamentales de l'analyse. Livre II: Algèbre. Chap. VI: Groupes et corps ordonnés, Actualitiés Sci. Indust., no. 1179, Hermann, Paris, 1952.

3. J. H. Grace, On the zeros of a polynomial, Proc. Cambridge Philos. Soc., 11 (19001902), 352-357.

4. E. Hille and R. S. Phillips, Functional analysis and semi-groups, rev. ed., Amer. Math. Soc. Colloq. Publ., Vol. 31, Amer. Math. Soc., Providence, R. I., 1957.

5. L. Hörmander, On a theorem of Grace, Math. Scand., 2 (1954), 55-64.

6. E. Laguerre, Oeuvres 1, Paris, 1898.

7. M. Marden, Geometry of polynomials, rev. ed., Math. Surveys, no. 3, Amer. Math. Soc., Providence, R. I., 1966.

8. — A generalization of a theorem of Bôcher, SIAM J. Numer. Anal., 3 (1966), $269-275$.

9. - On composite abstract homogeneous polynomials, Proc. Amer. Math. Soc., 22 (1969), 28-33.

10. G. Szegö, Bemerkungen zu einem Satz von J. H. Grace über die Wurzeln algebraischer Gleichungen, Math. Z., 13 (1922), 28-55.

11. B. L. Van der Waerden, Algebra, Vol. I, 4th ed., Die Grundlehren der math. Wissenschaften, Band 33, Springer-Verlag, Berlin, 1955; English transl., Ungar, New York, 1970.

12. J. L. Walsh, On the location of the roots of the Jacobian of two binary forms, and of the derivative of a rational function, Trans. Amer. Math. Soc., 22 (1921), 101116.

13. N. Zaheer, Null-sets of abstract homogeneous polynomials in vector spaces, Doctoral Thesis, Univ. of Wisconsin, Milwaukee, Wis., 1971.

14. On polar relations of abstract homogeneous polynomials, Trans. Amer. Math. Soc., 218 (1976), 115-131.

15. — On composite abstract homogeneous polynomials, Trans. Amer. Math. Soc., 228 (1977), 345-358. 
16. S. P. Zervos, Aspects modernes de la localisation des zéros des polynômes d'une variable, Ann. Sci. Ecole Norm Sup. (3) 77 (1960), 303-410.

Received February 15, 1977. Presented to the American Mathematical Society Jan. 18, 1972 under the title Null sets of abstract homogeneous polynomials in vector spaces; Received by the editors February 15, 1977. The results in this paper are partly contained in the author's doctoral dissertion (1971) at the University of Wisconsin-Milwaukee under the supervision of the University of Wisconsin-Milwaukee Distinguished Professor Morris Marden. The author is thankful to him for his helpful suggestions all along.

AligaRH MUSLIM UNIVERSITY,

ALIGARH-202001, INDIA 



\section{PACIFIC JOURNAL OF MATHEMATICS}

\section{EDITORS}

RICHARD ARENS (Managing Editor)

University of California

Los Angeles, CA 90024

Charles W. Curtis

University of Oregon

Eugene, OR 97403

C. C. Moore

University of California

Berkeley, CA 94720

\section{J. DugundJI}

Department of Mathematics

University of Southern California

Los Angeles, CA 90007

R. FInN and J. Milgram

Stanford University

Stanford, CA 94305

\section{ASSOCIATE EDITORS}
E. F. BECKENBACH
B. H. NeumanN
F. WOLF
K. YoSHIDA

\section{SUPPORTING INSTITUTIONS}

\author{
UNIVERSITY OF BRITISH COLUMBIA \\ CALIFORNIA INSTITUTE OF TECHNOLOGY \\ UNIVERSITY OF CALIFORNIA \\ MONTANA STATE UNIVERSITY \\ UNIVERSITY OF NEVADA, RENO \\ NEW MEXICO STATE UNIVERSITY \\ OREGON STATE UNIVERSITY \\ UNIVERSITY OF OREGON
}

\author{
UNIVERSITY OF SOUTHERN CALIFORNIA \\ STANFORD UNIVERSITY \\ UNIVERSITY OF HAWAII \\ UNIVERSITY OF TOKYO \\ UNIVERSITY OF UTAH \\ WASHINGTON STATE UNIVERSITY \\ UNIVERSITY OF WASHINGTON
}

The Supporting Institutions listed above contribute to the cost of publication of this Journal, but they are not owners or publishers and have no responsibility for its content or policies.

Mathematical papers intended for publication in the Pacific Journal of Mathematics should be in typed form or offset-reproduced, (not dittoed), double spaced with large margins. Please do not use built up fractions in the text of the manuscript. However, you may use them in the displayed equations. Underline Greek letters in red, German in green, and script in blue. The first paragraph or two must be capable of being used separately as a synopsis of the entire paper. Items of the bibliography should not be cited there unless absolutely necessary, in which case they must be identified by author and journal, rather than by item number. Manuscripts, in triplicate, may be sent to any one of the editors. Please classify according to the scheme of Math. Reviews, Index to Vol. 39. All other communications should be addressed to the managing editor, or Elaine Barth, University of California, Los Angeles, California, 90024.

50 reprints to each author are provided free for each article, only if page charges have been substantially paid. Additional copies may be obtained at cost in multiples of 50 .

The Pacific Journal of Mathematics is issued monthly as of January 1966. Regular subscription rate: $\$ 72.00$ a year (6 Vols., 12 issues). Special rate: $\$ 36.00$ a year to individual members of supporting institutions.

Subscriptions, orders for numbers issued in the last three calendar years, and changes of address should be sent to Pacific Journal of Mathematics, 103 Highland Boulevard, Berkeley, California, 94708. Older back numbers obtainable from Kraus Periodicals Co., Route 100, Millwood, NY 10546.

PUBLISHED BY PACIFIC JOURNAL OF MATHEMATICS, A NON-PROFIT CORPORATION

Printed at Kokusai Bunken Insatsusha (International Academic Printing Co., Ltd.). 8-8, 3-chome, Takadanobaba, Shinjuku-ku, Tokyo 160, Japan.

Copyright (C) 1978 by Pacific Journal of Mathematics

Manufactured and first issued in Japan 


\section{Pacific Journal of Mathematics

Vol. 74, No. $2 \quad$ June, 1978

Aharon Atzmon, Spectral synthesis in some spaces of bounded continuous

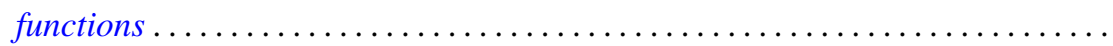

Karl Egil Aubert and Isidor Fleischer, Tensor products of ideal systems and their modules.............................................

Richard F. Basener, Several dimensional properties of the spectrum of a uniform

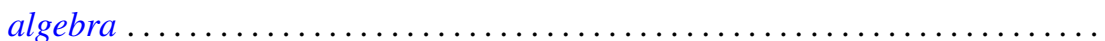

R. H. Bing and Michael Peter Starbird, Super triangulations ............. 307

Andrew Carson, Coherent polynomial rings over regular rings of finite index .....

Robert M. DeVos and Frederick W. Hartmann, Sequences of bounded summability domains .................................................

George Grätzer and R. Padmanabhan, Symmetric difference in abelian groups ....

Robert L. Griess, Jr., A remark about groups of characteristic 2-type and

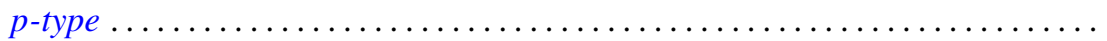

Emil Grosswald and F. J. Schnitzer, A class of modified $\zeta$ and L-functions........

Jutta Hausen and Johnny Albert Johnson, Ideals and radicals of some

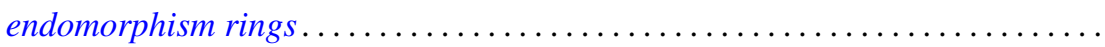

Jean Ann Larson, A solution for scattered order types of a problem of Hagendorf ............................................

Peter A. McCoy, Extremal properties of real biaxially symmetric potentials in $E^{2(\alpha+\beta+2)}$

Héctor Alfredo Merklen, Hereditary crossed product orders .

Hal G. Moore and Adil Mohamed Yaqub, Equational definability of addition in certain rings...

Robert Laurens Moore, Reductivity in $C^{*}$-algebras and essentially reductive operators. . .

Joseph Alvin Neisendorfer, Lie algebras, coalgebras and rational homotopy theory for nilpotent spaces...

William Raymond Nico, Bounded monoids

Richard Paul Osborne, Simplifying spines of 3-manifolds ...

Richard Paul Osborne, The simplest closed 3-manifolds. With an appendix by Osborne and J. Yelle.

Clayton Collier Sherman, The $K$-theory of an equicharacteristic discrete valuation ring injects into the $K$-theory of its field of quotients.... .

Mitchell Herbert Taibleson, The failure of even conjugate characterizations of $H^{1}$

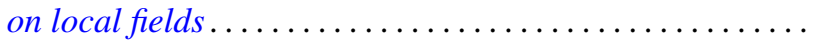

Keti Tenenblat, On characteristic hypersurfaces of submanifolds in Euclidean space ...................................

Jeffrey L. Tollefson, Involutions of Seifert fiber spaces..........

Joel Larry Weiner, An inequality involving the length, curvature, and torsions of a curve in Euclidean $n$-space .......................

Neyamat Zaheer, On generalized polars of the product of abstract homogeneous polynomials.... 